\title{
A novel approach to identify molecular binding to the influenza virus H5N1: screening using
} 5, 617 this: Med. Chem. Commun, 2014, molecularly imprinted polymers (MIPs) $\uparrow$

Thipvaree Wangchareansak, ${ }^{a}$ Arunee Thitithanyanont, ${ }^{b}$ Daungmanee Chuakheaw, ${ }^{a}$ M. Paul Gleeson, ${ }^{a}$ Peter A. Lieberzeit ${ }^{c}$ and Chak Sangma*a

In this report we investigate whether a molecularly imprinted polymer (MIP) of an inactivated strain of influenza A H5N1 could be used to help identify molecules capable of binding to, and inhibiting the function of the virus, via either competitive or allosteric mechanisms. Molecules which bind to the virus and induce a conformational change are expected to show reduced binding to the H5N1 specific MIP. Given the importance of molecular recognition in virus replication, such conformational change might also reduce the effectiveness of neuraminidase (N1) for cleaving the sialic groups necessary for virus replication. We show that the method can indeed differentiate between a potent neuraminidase inhibitor, $\mathrm{H} 1$ and $\mathrm{H} 5$ antibodies, and N1 specific and non-specific monosaccharide substrates. We suggest that such a method could potentially be used in conjunction with traditional biochemical assays to facilitate the identification of molecules functioning via novel modes of action.

Received 24th September 2013 Accepted 9th February 2014

DOI: $10.1039 / \mathrm{c} 3 \mathrm{md} 00272 \mathrm{a}$

www.rsc.org/medchemcomm inhibitors is that the methods used to assess binding are time and resource intensive.

In this paper we discuss the application of $\mathrm{H} 5 \mathrm{~N} 1$ influenza molecularly imprinted polymers (MIPs) to facilitate the identification of molecules that can induce sizeable changes in the target conformation on the virus surface such that it alters its structural recognition features. MIPs consist of a polymer matrix formed in the presence of a template. ${ }^{5,6}$ Once the template is removed, binding cavities remain that are highly selective to the template in question. MIPs can therefore be

probe the link between target modulation and efficacy. example the identification of non-competitive, allosteric inhibitors is a well known strategy, and methods that facilitate their identification are highly desirable.

Allosteric inhibitors, such as the non-nucleoside reversetranscriptase inhibitors (NNRTIs) of HIV1-RT, ${ }^{4}$ bind to a pocket adjacent to the orthosteric site occupied by the less effective 1st generation competitive nucleoside reverse-transcriptase inhibitors (NRTIs). NNRTI binding leads to a significant conformational change in the hetero-dimer structure as observed by X-ray crystallography and this prevents DNA replication from occurring. The key challenge in the identification of allosteric

${ }^{a}$ Department of Chemistry, Faculty of Science, Kasetsart University, Chatuchak, Bangkok, Thailand. E-mail: fscicsm@ku.ac.th; Fax: +66-2-5793955; Tel: +66-2-5625555-ext. 2192

${ }^{b}$ Department of Microbiology, Faculty of Science, Mahidol University, Rama 6 road, Phaya Thai, Bangkok, Thailand

${ }^{c}$ Department of Analytical Chemistry, University of Vienna, Vienna, Austria

† Electronic supplementary information (ESI) available. See DOI: $10.1039 / \mathrm{c} 3 \mathrm{md} 00272 \mathrm{a}$

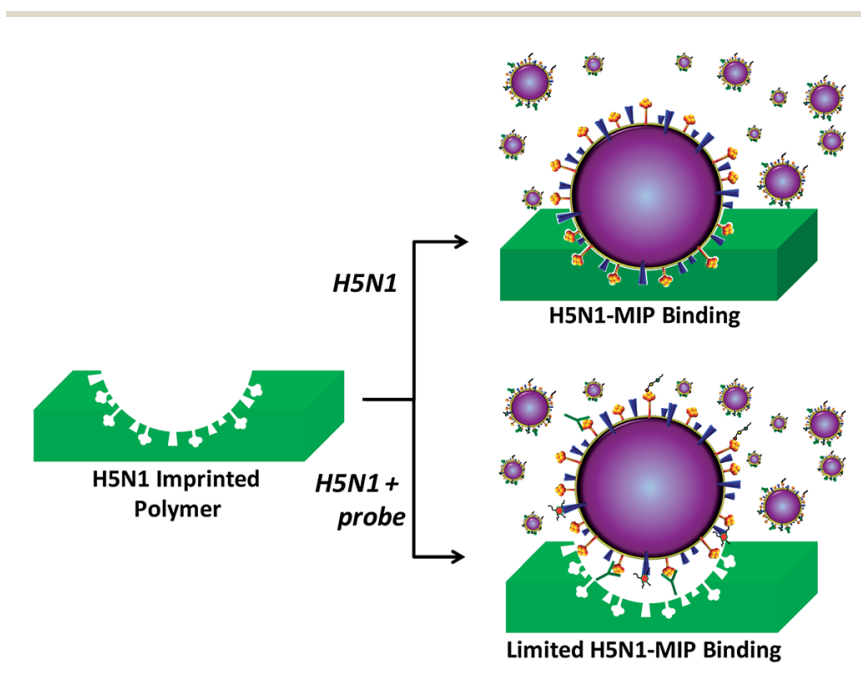

Fig. 1 Schematic of H5N1 virus binding to a MIP (top) and inhibition of the process due to a conformational change in the H5N1-probe complex. 
used to selectively identify specific chemicals, proteins or virus particles, ${ }^{7,8}$ and their concentration., ${ }^{9,10}$

In Fig. 1, we show a schematic representation of the H5N1 virus binding to its corresponding MIP. H5N1 has two major proteins on its surface, hemagglutinin (HA) and neuraminidase (NA). HA binds to a host cell receptor when the virus is initially entering the host cell. NA, then, cleaves the terminal group of sialic acid from the cell receptor and pushes itself into the inner cell. The binding of a probe molecule(s) to the virus particle will interrupt binding to the MIP (bottom of Fig. 1) if it induces a sufficiently large conformational change. Therefore, recognition is expected to show concentration dependence, while the use of a control system to take into account non-specific molecular binding to the template is also employed.

\section{Results and discussion}

Recently, we have succeeded in influenza A virus imprinting where the MIP was able to discriminate between virus subtypes using a quartz crystal microbalance (QCM).${ }^{11}$ The QCM method relies on the principle that the resonance frequency of a QCM in an oscillator will change depending on the mass removed from or added to the quartz surface. The relationship between mass and frequency can be determined by the Sauerbrey equation: ${ }^{12}$

$$
\Delta f=-c \Delta m
$$

where $c$ is a constant value determined by the sensitive area and fundamental frequency. We have coated a QCM electrode with an influenza virus MIP and used it to measure the recognition of a virus sample in terms of frequency change. The results suggest that recognition of a unique pattern of the specific area by the MIP is possible. The QCM can differentiate between influenza A subtypes which have identical structures but different numbers of distinctive amino acids on the surface.

In this work, we try to confirm this hypothesis by using a similar experiment to monitor absorption of the H5N1 virus before and after reactions with molecules known to bind to H5N1 at different sites (Fig. 1). According to this scheme, the virus absorption on the H5N1 MIP should decrease when a probe molecule is attached to the virus surface, thereby preventing it from binding to the MIP. In this experiment, the probe molecules and their binding sites on the influenza A virus subjected to this experiment are anti-H5 and anti-H1 antibodyinfluenza A hemagglutinin (HA) antibodies, sialic acid and $N$-acetylglucosamine (GlcNAc) derivatives ${ }^{13}$ - parts of the influenza A receptor targeting to the HA protein at the receptor binding pocket, and oseltamivir - an anti-neuraminidase drug.

The influenza A based MIP used in this experiment was prepared as a co-polymer using acrylamide (AAM), methacrylic acid (MAA), methylmethacrylate (MMA) and $N$-vinylpyrrolidone (VP) based on our previously reported method. ${ }^{11}$ The polymer surface was stamped using an inactivated virus template (H5N1).

Our MIP uses not one, but four monomers with different side-chains during preparation. These side-chains allow the MIP to have both polar and hydrophobic functionalities with which to interact with protein surfaces. Imprinting generates recognition sites for molecules or microorganisms in synthetic polymers by adding a template to the respective reaction mixture just before polymerization. The monomers still have the possibility of aligning themselves around the template where they can pre-form a specific non-covalent interaction network. After hardening and removal of the template, the polymer surface of the MIP retains information of the template's shape, size and the surface properties induced by selforganization. ${ }^{14}$ This explains how minute details are detectable and how a decrease in QCM signal can occur as a result of molecular binding.

The ability of the MIP to absorb H5N1 was subsequently measured using a QCM device as illustrated in Fig. 2. The detection limit of the assay was found to be equivalent to 1 HA titer unit (HAU). In subsequent screening applications, all of the experiments used the following procedure. First, the probe molecules at various concentrations were mixed with fixed concentration of H5N1 virus (8 HAU). Each mixture was injected into the QCM measuring cell (containing both MIP and nonimprinted polymer (NIP)) and the relative frequency change was recorded (see Experimental section for details). After each experiment the absorbed H5N1 particles were washed off the surface of the MIP. Each experiment was performed in triplicate.

To assess the ability of the MIP approach to differentiate between molecular binders of different types and affinities, we used the following probes: H5 antibody ${ }^{15}$ (high affinity), oseltamivir (high affinity), ${ }^{16}$ sialic acid (Sia) ${ }^{17}$ (moderate affinity), GlcNAc $^{13}$ (low affinity) and H1 antibody (low affinity).

The probes used in this study were of different size, shape, and binding affinity. Sia and GlcNAc are constituents of the pentasaccharide, Sia-Gal-GlcNAc-Gal-Glc (linkage-type omitted), which is one of the cell receptors of influenza A. ${ }^{18,19} \mathrm{Sia}$ is the terminal portion of the molecule that enters into the Sia binding pocket of HA while the GlcNAc substructure is located outside of the binding pocket. The latter is therefore expected to be of low overall affinity without the attached Sia head group. ${ }^{17}$

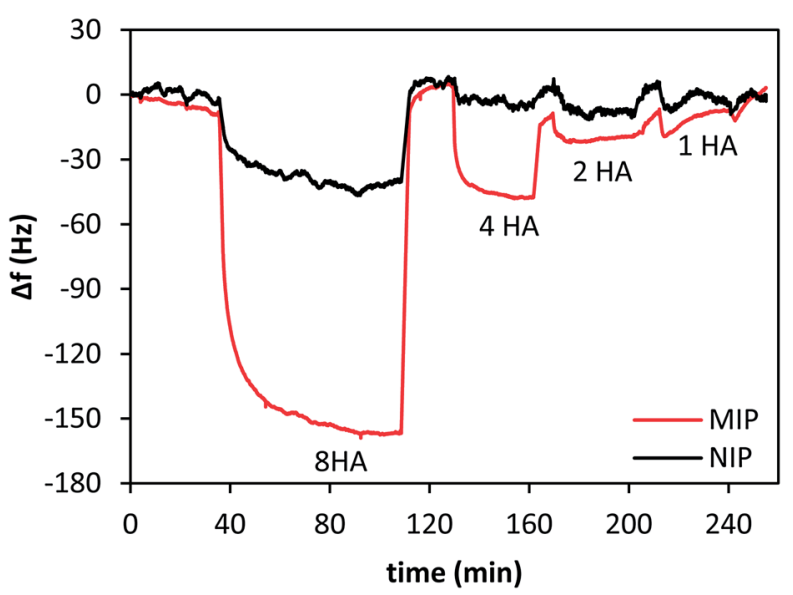

Fig. 2 H5N1 MIP QCM measurements using the MIP (red), and NIP (black) with differing concentrations of H5N1. More extensive binding leads to a larger frequency change between the MIP and the reference NIP. 
In Fig. 3, the results obtained for the high and moderate affinity probes are reported, while those for the low affinity probes are shown in Fig. 4. Firstly, it should be noted that the resolution of the MIP towards large macroscopic virus particles is clearly lower than for small molecules. ${ }^{20}$ This can be seen in Fig. 3 where we were unable to fully prevent the binding of $\mathrm{H} 5 \mathrm{~N} 1$ to the MIP, even at the highest probe concentrations. We propose that this is due to the fact that binding to the MIP can occur via different surfaces of the virus and some of these may be slightly less susceptible to conformational changes upon probe binding. This is in line with previous experiments with erythrocytes, where different glycolipid surface concentrations even led to subgroup selectivity with a MIP ${ }^{21}$ or other virus serotype selectivity, as shown
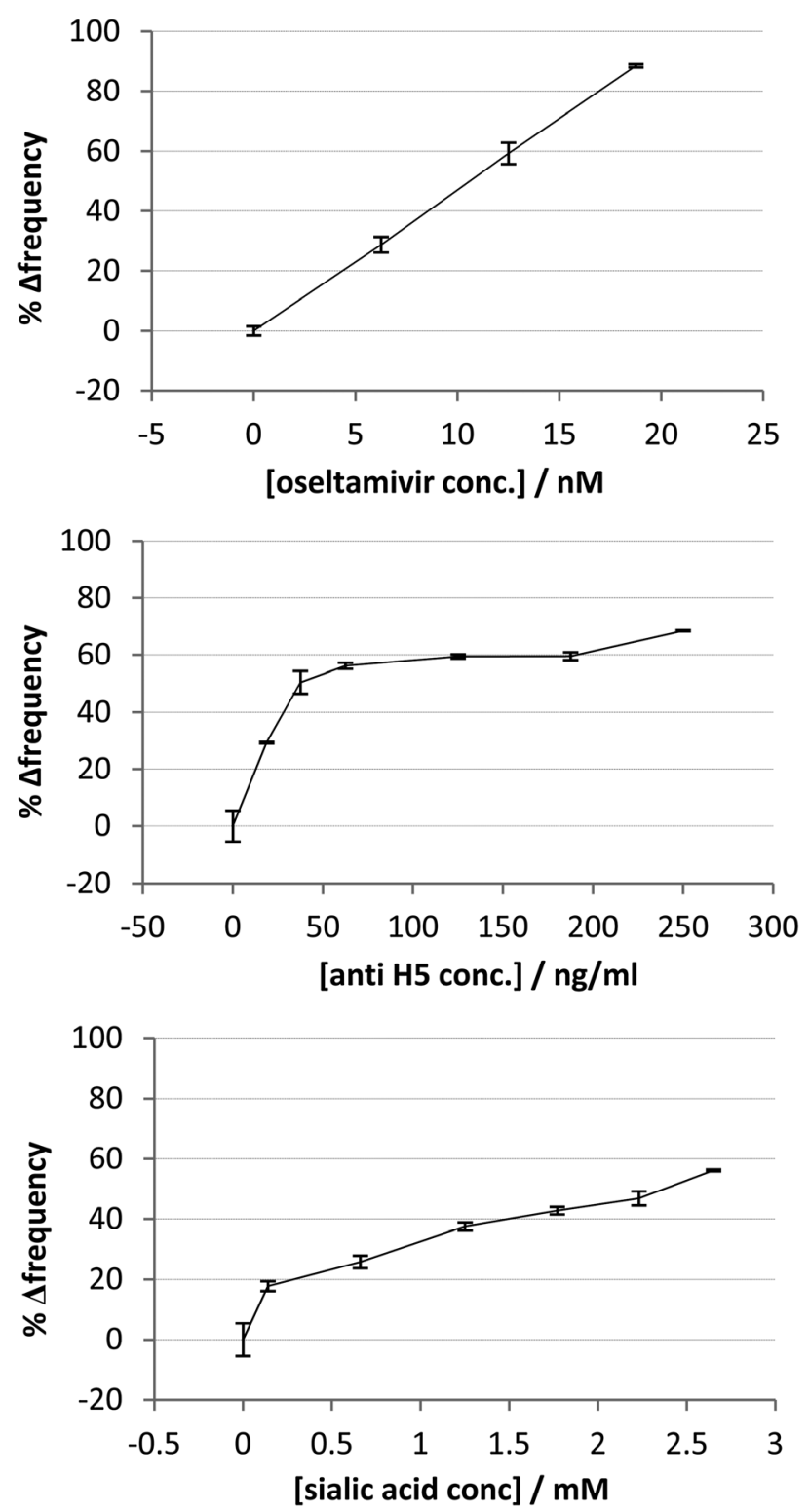

Fig. 3 The effects of probe modified H5N1 binding to the H5N1 based MIP. The greater the affinity of the probe for H5N1, the lower the binding as given by $\Delta$ freq with respect to $\mathrm{H} 5 \mathrm{~N} 1$ alone. All QCM experiments were carried out at $25^{\circ} \mathrm{C}$.
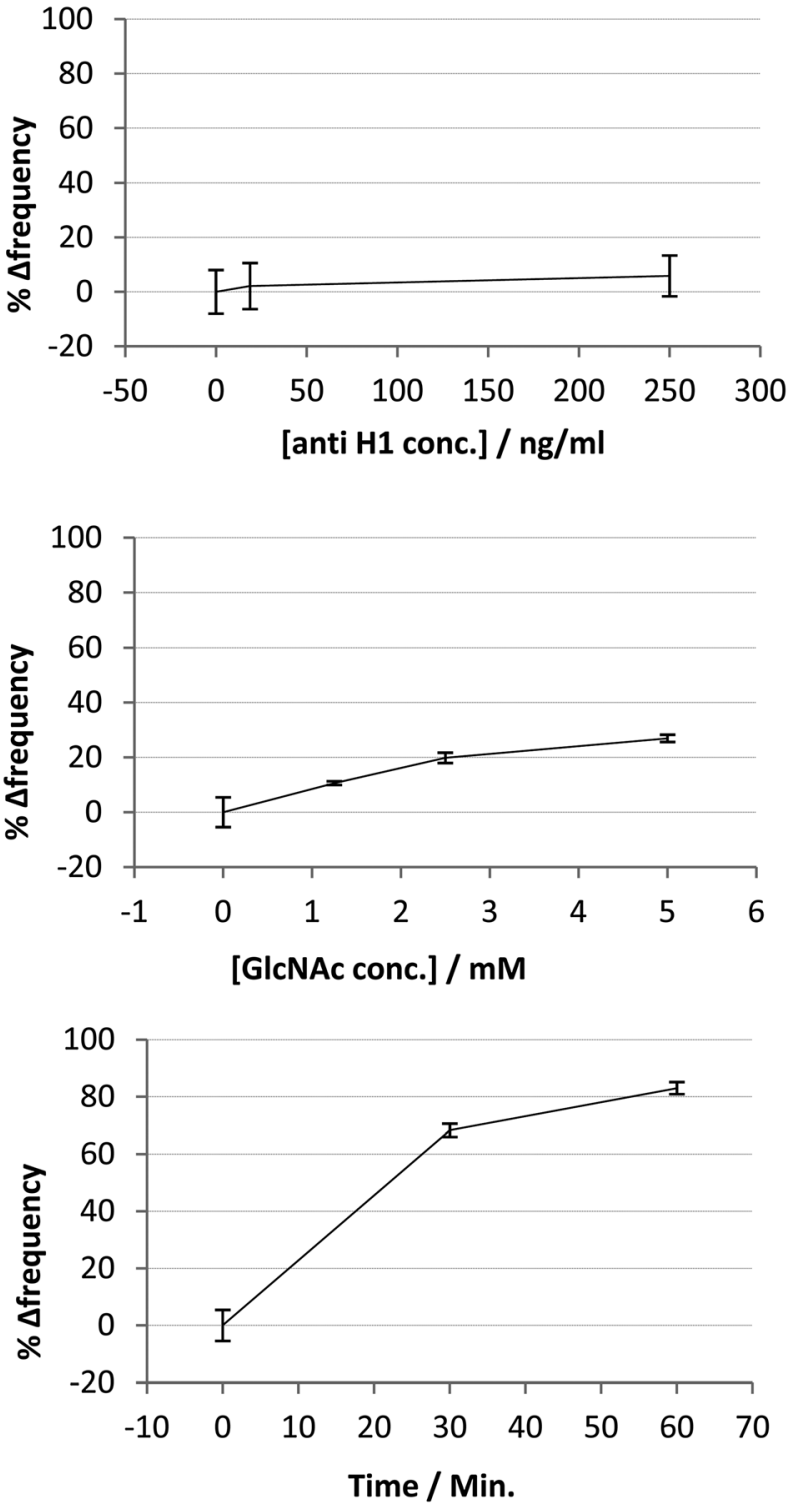

Fig. 4 The effects of probe modification and temperature on $\mathrm{H} 5 \mathrm{~N} 1$ binding to the H5N1 based MIP. This figure shows the results from the low affinity probes: non-specific binding probe (anti-H1, top); (GlcNAc, middle), and temperature effect (bottom) (by time used to heat the virus sample to $90^{\circ} \mathrm{C}$ ) for $\mathrm{H} 5 \mathrm{~N} 1$ binding samples. All QCM experiments were carried out at $25^{\circ} \mathrm{C}$.

with human rhinovirus (HRV). ${ }^{7}$ Nevertheless, given that high potency oseltamivir ( $\left.\mathrm{IC}_{50} \sim 1 \mathrm{nM}\right)^{22}$ more strongly inhibits the binding of $\mathrm{H} 5 \mathrm{~N} 1$ to the polymer than anti-H5 $\left(\mathrm{IC}_{50} \sim 5-34 \mathrm{ng}\right.$ $\left.\mathrm{ml}^{-1}\right),{ }^{23}$ which is in turn more effective than sialic acid (3$10 \mathrm{mM}){ }^{24}$ the method is clearly capable of distinguishing between ligands of different affinities and type. Crudely, the points where $50 \%$ response was reached in the MIP based experiments were approximately $10 \mathrm{nM}, 36 \mathrm{ng} \mathrm{ml} \mathrm{m}^{-1}$ and $2.4 \mathrm{mM}$ respectively which is in reasonable agreement with the experimental values. Furthermore, analysis of the results for the $\mathrm{H} 1$ antibody (Fig. 4) show a maximum response at $\sim 10 \%$ while that for the low affinity substrate (GlcNAc) reaches $\sim 25 \%$ at the highest concentrations. 
We use a separate control experiment to confirm that the signals observed came from H5 absorption and not other binding agents. For this purpose, heat was applied to denature the viral protein, and it was observed that this reduces the signal to approximately $50 \%$ of its initial value due to denaturing (Fig. 4). In addition, we observe a much smaller and nonspecific response in QCM signal from probe binding to the NIP and MIP, suggesting that each probe does indeed bind to the virus to limit binding to the MIP (see ESI $\dagger$ ).

These results suggests that the method is clearly capable of differentiating between induced conformational effects from small inhibitors and substrates, to large macro-molecules and could certainly be used as a rapid screening tool to estimate the approximate binding affinity and whether binding results in a noticeable conformational effect (otherwise the method will not provide discrimination). Furthermore, to the very best of our knowledge this is one of the first attempts to actually characterize the effect of drugs on their target bio-species in situ.

\section{Experimental}

\section{Virus preparation}

H5N1 (A/open-billed stork/Nakhonsawan/BBD0104F/04) was isolated from an open-billed stork and propagated in MDCK at P3 biosafety laboratory, Department of Microbiology, Mahidol University, Bangkok, Thailand. In this experiment we used only inactivated virus and carried out all experiments in a strictly controlled laboratory.

\section{Virus imprinting}

The virus imprinting protocol and copolymer conditions followed our previous work. ${ }^{11}$ Briefly, the polymer system consisted of acrylamide $(13.0 \mathrm{mg})$, methacrylic acid (10.6 mg), methylmethacrylate $(6 \mathrm{mg})$, and $N$-vinylpyrrolidone $(6.3 \mathrm{mg})$ dissolved in $300 \mu \mathrm{l}$ of dimethylsulfoxide containing the initiator $2,2^{\prime}$-azobi$\mathrm{s}$ (isobutyronitrile). Afterwards, this was pre-polymerized at $70{ }^{\circ} \mathrm{C}$ for 40 minutes to reach a gel point. These pre-polymerization polymers were dropped on to the QCM electrode and spun off to obtain a thin layer. The template stamp was prepared on a glass substrate by adding $5 \mu \mathrm{l}$ of the H5N1 virus sample and kept at $4{ }^{\circ} \mathrm{C}$ for 30 minutes. Then, the template stamp was pressed onto the polymer layer and polymerized under UV light $(254 \mathrm{~nm})$ overnight. The imprinted cavities of the H5N1 virus were obtained by removing the virus template from the rigid polymer with $10 \%$ hydrochloric acid and stirred in water at $45{ }^{\circ} \mathrm{C}$ for 3 hours. Finally, the H5N1 imprinted polymer was installed into the measuring cell for use in QCM measurements.

\section{QCM measurements}

The QCM with dual gold electrodes was placed into the custommade poly(dimethylsiloxane) cell (75 $\mu$ l volume). This measuring cell was connected to an oscillator circuit and a frequency counter (Agilent 53131A) for real-time frequency read-out from a computer via GPIB interface and LabView software. The QCM experiments measured 2 channels at the same time at $25{ }^{\circ} \mathrm{C}$. It was started by injecting the PBS buffer
(pH 7.2) into the measuring cell to obtain a baseline. After the frequency change in both channels reached a stable value, the PBS was replaced with the H5N1 sample and left to reach equilibrium. This was followed by washing with $10 \%$ acetic acid, $3 \times$ water and $3 \times$ PBS buffer prior to measurements being restarted. After the frequency had returned to its initial value, the cell was ready for the next sample.

\section{Virus binding assay}

The molecules used in the virus binding assay experiments were CMP-sialic acid (Acros), $p$-nitrophenyl- $N$-acetylglucosamine (Sigma-Aldrich), oseltamivir (obtained from the Thailand Government Pharmaceutical Organization), antibody $\mathrm{H} 5$ and antibody $\mathrm{H} 1$ (Immune Tech). For each binding assay, we collected QCM signals from different ligand/antibody concentrations at fixed H5N1 concentration (8 HAU) using the same procedure for QCM measurement. For binding assay samples, we prepared the samples by mixing $8 \mathrm{HAU}$ of $\mathrm{H} 5 \mathrm{~N} 1$ with the chosen molecule (such as an antibody or drug) in a small tube then left it for 30 minutes for the binding process to take place. Then, this sample mixture was ready to use for QCM measurement. Heat was used as the negative control to denature the virus. The virus samples were heated to $90{ }^{\circ} \mathrm{C}$ for 30 minutes and 90 minutes, before being left to cool down to room temperature. Then, this virus sample was injected into the measuring cell and the signal change was recorded.

\section{Conclusions}

We have used a MIP based approach to screen molecular probes of differing size and affinity for H5N1. We find that H5N1-based MIPs can successfully differentiate between high and low affinity probe molecules due to the conformational changes induced by binding. This conformational change reduces the affinity of the H5N1-probe complex for the MIP compared with using unmodified H5N1. The net reduction in binding, as given by the weight of virus bound to the MIP, is proportional to their known binding constants. These initial results suggest that a MIP based methodology could be used to screen for inhibitors capable of inducing conformational change in the target protein. While the resolution of the process is somewhat limited by the fact that the MIP recognition sites may be formed by surfaces unchanged by probe binding, it is possible with the diverse probes used here to see an effect proportional to their know affinities. Such a method could prove useful for other proteins such as HIV1-RT or EGFR where binding within an allosteric pocket/region is known to produce a sizeable conformational change in the protein structure and confer a considerable therapeutic advantage over nonallosteric inhibitors.

\section{Acknowledgements}

The authors thank the RGJ-PhD program, Thailand Research Fund DBG5280004, ASEA-UNINET, Kasetsart National Research University Project, Kasetsart University Research and Development Institute for financial support of this work. We also thank 
S. Hannongbua, from Chulalongkorn University; S. Hannongbua and J. Limtrakul, from Kasetsart University, Thailand; and P. Wolschann, F. L. Dickert and F. M. Unger from the University of Vienna for their support of this study. We thank R. G. Webster and R. J. Webby for the 8-plasmid reverse genetics system.

\section{Notes and references}

1 R. Macarron, M. N. Banks, D. Bojanic, D. J. Burns, D. A. Cirovic, T. Garyantes, D. V. Green, R. P. Hertzberg, W. P. Janzen, J. W. Paslay, U. Schopfer and G. S. Sittampalam, Nat. Rev. Drug Discovery, 2011, 10, 188195.

2 A. Fernan, S. R. Burrows, D. J. Moss, A. Saul and A. Suhrbier, Immunol. Lett., 1991, 30, 339-344.

3 P. J. Hajduk and D. J. Burns, Comb. Chem. High Throughput Screening, 2002, 5, 613-621.

4 M. Lapkouski, L. Tian, J. T. Miller, S. F. Le Grice and W. Yang, Nat. Struct. Mol. Biol., 2013, 20, 230-236.

5 B. Sellergren, Anal. Chem., 1994, 66, 1578-1582.

6 S. A. Piletsky, N. W. Turner and P. Laitenberger, Med. Eng. Phys., 2006, 28, 971-977.

7 M. Jenik, R. Schirhagl, C. Schirk, O. Hayden, P. Lieberzeit, D. Blaas, G. Paul and F. L. Dickert, Anal. Chem., 2009, 81, 5320-5326.

8 L. D. Bolisay, J. N. Culver and P. Kofinas, Biomaterials, 2006, 27, 4165-4168.

9 F. L. Dickert, O. Hayden, R. Bindeus, K. J. Mann, D. Blaas and E. Waigmann, Anal. Bioanal. Chem., 2004, 378, 1929-1934.

10 M. A. Cooper, Y. Uludag, S. A. Piletsky and A. P. F. Turner, FEBS J., 2007, 274, 5471-5480.

11 T. Wangchareansak, A. Thitithanyanont, D. Chuakheaw, M. P. Gleeson, P. A. Lieberzeit and C. Sangma, J. Mater. Chem. B, 2013, 1, 2190.

12 G. Sauerbrey, Zeitschrift für Physik, 1959, 155, 206-222.
13 T. Wangchareansak, C. Sangma, P. Ngernmeesri, A. Thitithanyanont and P. A. Lieberzeit, Anal. Bioanal. Chem., 2013, 405, 6471-6478.

14 L. X. Chen, S. F. Xu and J. H. Li, Chem. Soc. Rev., 2011, 40, 2922-2942.

15 T. Rowe, R. A. Abernathy, J. Hu-Primmer, W. W. Thompson, X. H. Lu, W. Lim, K. Fukuda, N. J. Cox and J. M. Katz, J. Clin. Microbiol., 1999, 37, 937-943.

16 M. D. de Jong, T. T. Tran, H. K. Truong, M. H. Vo, G. J. Smith, V. C. Nguyen, V. C. Bach, T. Q. Phan, Q. H. Do, Y. Guan, J. S. Peiris, T. H. Tran and J. Farrar, N. Engl. J. Med., 2005, 353, 2667-2672.

17 C. C. Wang, J. R. Chen, Y. C. Tseng, C. H. Hsu, Y. F. Hung, S. W. Chen, C. M. Chen, K. H. Khoo, T. J. Cheng, Y. S. Cheng, J. T. Jan, C. Y. Wu, C. Ma and C. H. Wong, Proc. Natl. Acad. Sci. U. S. A., 2009, 106, 18137-18142.

18 J. Stevens, O. Blixt, T. M. Tumpey, J. K. Taubenberger, J. C. Paulson and I. A. Wilson, Science, 2006, 312, 404410.

19 Y. Ha, D. J. Stevens, J. J. Skehel and D. C. Wiley, Proc. Natl. Acad. Sci. U. S. A., 2001, 98, 11181-11186.

20 D. R. Kryscio and N. A. Peppas, Acta Biomater., 2012, 8, 461473.

21 A. Seifner, P. Lieberzeit, C. Jungbauer and F. L. Dickert, Anal. Chim. Acta, 2009, 651, 215-219.

22 D. A. Boltz, B. Douangngeun, P. Phommachanh, S. Sinthasak, R. Mondry, C. Obert, P. Seiler, R. Keating, Y. Suzuki, H. Hiramatsu, E. A. Govorkova and R. G. Webster, J. Gen. Virol., 2010, 91, 949-959.

23 H. Hu, J. Voss, G. Zhang, P. Buchy, T. Zuo, L. Wang, F. Wang, F. Zhou, G. Wang, C. Tsai, L. Calder, S. J. Gamblin, L. Zhang, V. Deubel, B. Zhou, J. J. Skehel and P. Zhou, J. Virol., 2012, 86, 2978-2989.

24 J. J. Skehel and D. C. Wiley, Annu. Rev. Biochem., 2000, 69, 531-569. 